Supplement of Geosci. Model Dev., 10, 2891-2904, 2017

https://doi.org/10.5194/gmd-10-2891-2017-supplement

(C) Author(s) 2017. This work is distributed under

the Creative Commons Attribution 3.0 License.

(c) (1)

Supplement of

\title{
GNAQPMS v1.1: accelerating the Global Nested Air Quality Prediction Modeling System (GNAQPMS) on Intel Xeon Phi processors
}

Hui Wang et al.

Correspondence to: Zifa Wang (zifawang@mail.iap.ac.cn)

The copyright of individual parts of the supplement might differ from the CC BY 3.0 License. 


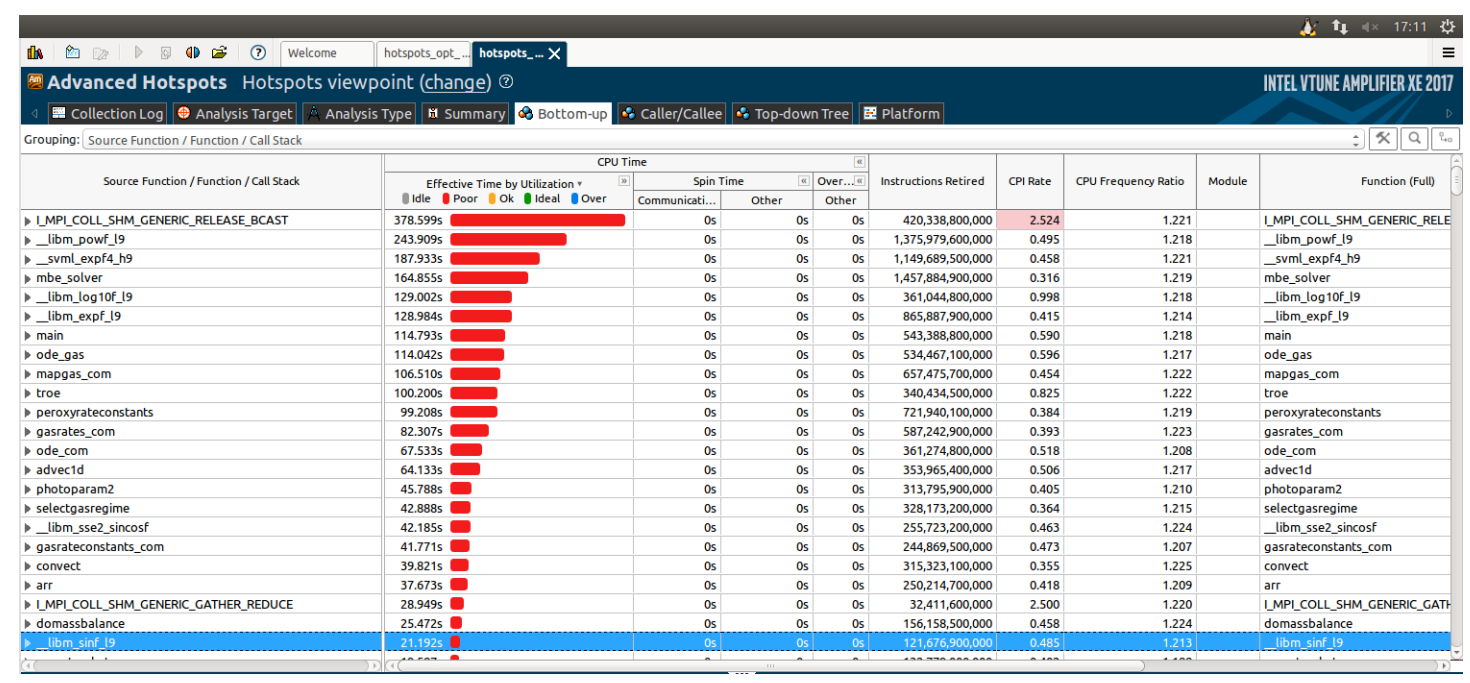

Figure S1. The hotspot functions detected by Vtune in Base-V GNAQPMS. The red bar shows the low CPU utilization of the functions in Base-V GNAQPMS, which could be optimized to improve the code performance.

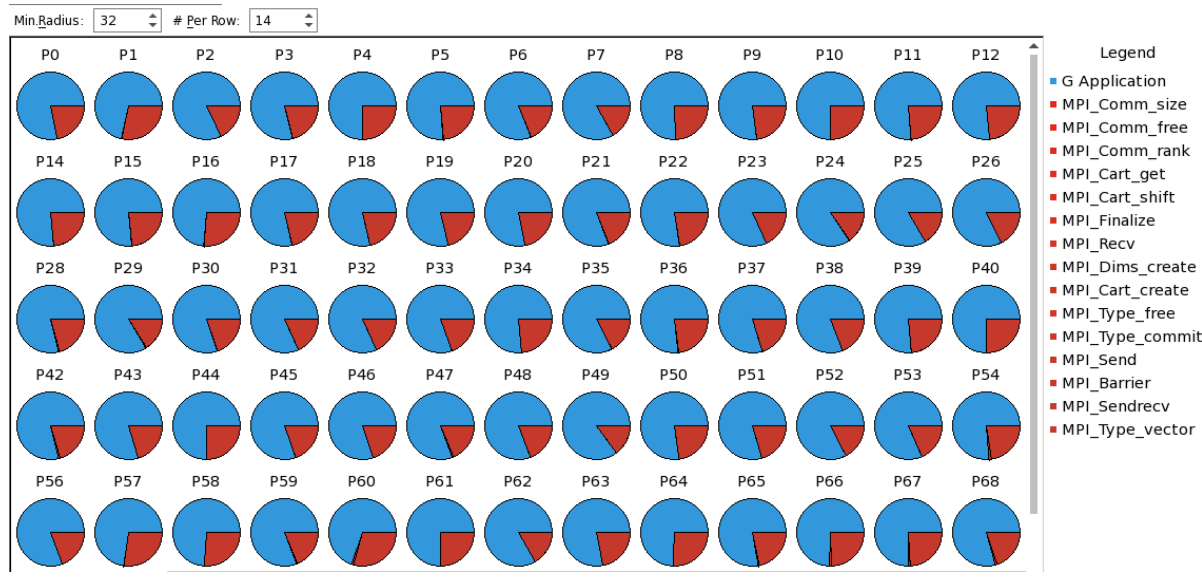

Figure S2. Load balance situation of GANQPMS on single CPU nodes. The pie figures shows the contributions from MPI and application codes for the GNAQPMS on the MPI processes. The almost equal blues parts indicate that the load balance is not the bottleneck of Base-V GNAQPMS. 


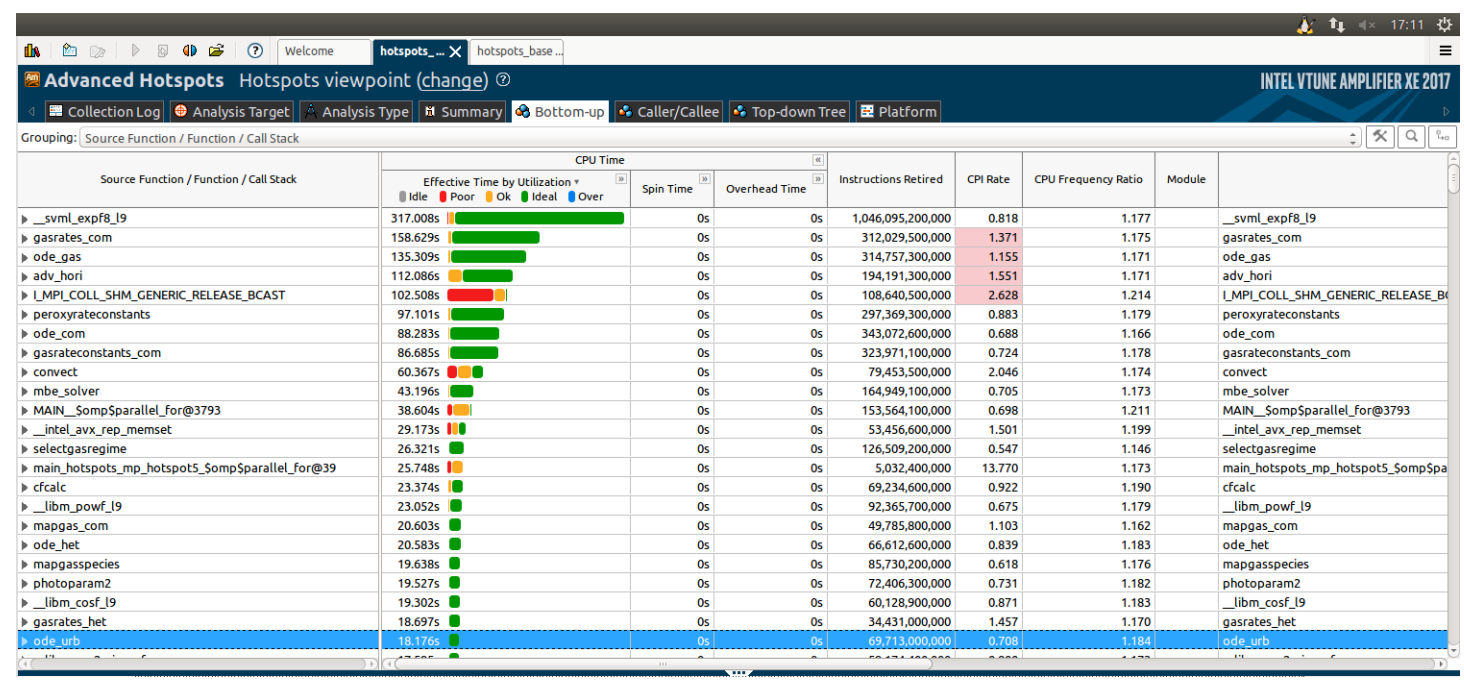

Figure S3. Hotspot functions detected by Vtune in Opt-V GNAQPMS. The green bars indicate the high CPU utilization directly. 